\title{
Identification of normal and pathological posterior inter-malleolar ligament with dedicated high-field vs low-field MRI. A pilot study
}

\author{
Raffaello Sutera ${ }^{1}$ \\ Antonino Bianco ${ }^{2}$ \\ Antonino Paoli ${ }^{3}$ \\ Johnny Padulo 4,5 \\ Ewan Thomas ${ }^{2}$ \\ Angelo lovane ${ }^{2}$ \\ Antonio Palma ${ }^{2}$
}

1 DIBIMEF, Section of Radiological Sciences, University of Palermo, Italy

2 Sport and Exercises Research Unit, University of Palermo, Italy

${ }^{3}$ Department of Biomedical Science, University of Padua, Italy

4 University eCampus, Novedrate, Italy

5 Tunisian Research Laboratory "Sports Performance Optimization" National Center of Medicine and Science in Sport, Tunis, Tunisia

Corresponding author:

Johnny Padulo

University eCampus

Via Isimbardi, 10

22060 Novedrate, Italy

E-mail: sportcinetic@gmail.com

\section{Summary}

Aim: the aim of the study was to determine an objective measure of detection of posterior inter-malleolar ligament (PIML) through a magnetic resonance (MRI) of the ankle with two dedicated scanners: high-field (1-Tesla: HMF) and low-field (0.2-Tesla: LMF).

Methods: two-hundred subjects were randomly recruited for the study and then divided in two groups (HMF and LMF). We retrospectively evaluated the MRI of the ankle in the two groups of patients. PIML evaluation was performed globally and separately using different scan planes.

Results: in HMF and LMF, the PIML was identified respectively in 55 and $11 \%$ of cases. PIML was classified as "indeterminate" in $\mathbf{2 8}$ and $\mathbf{5 7 \%}$ of patients, and "absent" in 17 and $32 \%$ of patients. In HMF and LMF the isolated evaluation on the coronal, axial and sagittal planes allowed PIML identification respectively in 100 and $100 \%, 67.27$ and $45.45 \%, 45.45$ and $12.4 \%$ of cases. In 5 cases $(4 / 5$ of HMF) we also observed a posterior ankle im- pingement syndrome (PAIS) determined by the PIML, with ligament changes (5/5) and associated synovial reactions (1/5), and an arthroscopic confirmation was obtained in $3 / 5$ cases.

Conclusion: the presence of the PIML seems to be a possible cause of PAIS and the use of a high-field MR scanner seems optimal for its identification.

KEY WORDS: ankle, ligament, magnetic resonance, musculoskeletal.

\section{Introduction}

In scientific literature the diagnostic evaluation of posterior ankle ligamentous compartment usually describes and represents ichonographically only two major ligaments, the posterior inferior tibio-fibular (PITFL) and the posterior talo-fibular ligament (PT$\mathrm{FL})^{1,2}$. Although, some authors describe a third welldefined ligamentous structure, independent and distinct from the PTFL and PITFL, the posterior intermalleolar ligament $(P I M L)^{1,3,4}$. This ligamentous structure is located between the posterior transverse ligament, the deepest and lower component of the PITFL, and the PTF $1,3,4$. The PIML runs from the top edge of the fibular malleolar fossa to the posterior edge of the tibial malleolus ${ }^{4}$.

From a review of the literature we noted some discrepancies either on the anatomy of $\mathrm{PIML}^{1}$ (in some cases misunderstood or confused with other ligamentous structures), either on its nomenclature, so that often the PIML has also been reported as "tibial slip"5,6 or as "marsupial meniscus", discrepancy is the clinical relevance of the PIML that is to some extent controversial as stated by Golanò et al. ${ }^{1}$. Only a few authors attribute a role to the PIML among the possible causes of the posterior ankle impingement syndrome (PAIS) ${ }^{1,8-10}$. Some recent arthroscopic and anatomical studies performed on sections of cadavers have shown that the PIML is present in the majority of the population, with a reported incidence ranging between 72.5 and $100 \%$, and so represents a normal variant of ligamentous anatomy of the ankle $1,3,11$. In the literature the normall $^{1,3}$ and pathological appearance of the PIML ${ }^{9}$ using high-field non specific MRI equipment with dedicated coils for the ankle has been well described. The PIML is a ligament of the posterior compartment of the ankle. There are some controversial issues in literature in regard to this ligament: 1) its independence 
Identification of normal and pathological posterior inter-malleolar ligament with dedicated high-field vs low-field MRI. A pilot study

from other anatomical ligamentous structures, such as the PITFL or PTFL ${ }^{1}$, and 2) there is difference between it and the tibial slip, definition with which it is identified by some authors ${ }^{1,5}$. As reported by $\mathrm{Oh}$ et al. $^{3}$ and Morgan ${ }^{12}$ there is a clear difference between PIML and the tibial slip, which therefore identify them as two different anatomical structures. Contrary to what stated by the above mentioned authors, we do not share their opinion, and we believe that they represent the same ligament, as said by Stoller et al. ${ }^{5}$ and Golanò et al. ${ }^{1}$. The MRI appearance of PIML has been described in a variety of anatomical, arthroscopic and radiological studies that reported several incidences of identification ${ }^{1,3,4}$.

Therefore, we carried out a pilot study about the identification of normal and pathological posterior intermalleolar ligament with dedicated high-field vs lowfield MRI.

\section{Materials and methods}

\section{Subjects}

A case-control study was carried out. We retrospectively and randomly searched within the picture archiving and communication system (PACS) of the Institute of Radiology of the University of Palermo a total of 200 subjects which underwent ankle MRI examination with dedicated scanners in a period between January and October 2013. The control group (HMF) was composed by 100 patients randomly recruited. On the other side, the experimental group (LMF), was also randomly recruited until a total number of 100 patients. In HMF group there were 58 men and 42 women age ranging between 11 and 56 years (mean age: $36.4+/-15 \mathrm{SD}$ ); height range between 154 and $197 \mathrm{~cm}$ (mean height 172 +/- 12 SD); weight range between 48 and $106 \mathrm{~kg}$ (mean weight: 76.4 +/20 SD). In LMF group there were 55 men and 45 women with age ranging between 13 and 58 years (mean age: $35.2+/-16 \mathrm{SD}$ ); height range between 148 and $196 \mathrm{~cm}$ (mean height of 169 +/- 13 SD); weight range between 50 and $104 \mathrm{~kg}$ (mean weight: 75.6 +/- 19 SD). The two groups showed no significant epidemiological differences. In our research approach, we considered the HMF MRI scanner as our Gold Standard. The opinion of the ethics committee was not required, since the evaluation of the MRI examination was performed retrospectively and in con- sideration of compliance with the rules of the Helsinki Declaration, the MRI examination was performed for diagnostic purposes in well-established and standardized modalities according to the common clinical procedure. The manuscript abides the ethical standards of the Muscles and Ligaments Journal ${ }^{13}$.

\section{Procedures}

In order to verify the identifiability of PIML we examined the MRI of the ankle of two groups of patients: HMF and LMF. The parameters used for MRI examination in the control group, with a dedicated HMF MRI scanner (Orthone, Oni Medical Systems, Wilmington, MA, USA), were: field of view (FOV) ranging between 12 and $14 \mathrm{~cm}$, reconstruction matrix of $256 \times 192$, slice thickness of $4 \mathrm{~mm}$ without reconstruction interval.

We performed STIR (TR/TE/TI 4700/12/160 ms) and FSE-T1 sequences (TR/TE 525/15 ms) in the sagittal plane, FSE-T2 sequences (TR/TE $3000 / 80 \mathrm{~ms}$ ) in the axial plane, and STIR (TR/TE/TI $4700 / 12 / 160 \mathrm{~ms}$ ) or GE-2D (TR/TE 550/18 ms, FA $25^{\circ}$ ) or FSE-T2 sequences (TR/TE $3000 / 80 \mathrm{~ms}$ ) in the coronal plane. The parameters used for MRI examination in the experimental group, with a dedicated LMF MRI scanner (Artoscan-C, Esaote, Genoa, Italy), were: FOV ranging between 12 and $14 \mathrm{~cm}$, reconstruction matrix variable between $192 \times 90$ and $256 \times 102$, slice thickness of $4 \mathrm{~mm}$ without reconstruction interval. We performed STIR (TR/TE/TI 1320/14/50 ms) and SE-T1 sequences (TR/TE 600/16 ms) in the sagittal plane, STIR sequences (TR/TE $3000 / 80 / 50 \mathrm{~ms}$ ) in the axial plane, SE-T2 (TR/TE 2300/90 ms) and GE-T2 sequences (TR/TE 900/20/60 ms) in the coronal plane.

\section{Diagnostic evaluation}

Two radiologists with more than 8 years of experience in musculoskeletal MRI re-evaluated the presence or absence of PIML, referring to the results obtained by Oh et al. ${ }^{3}$ who describe the identification of the PIML in different scanning planes (coronal, axial and sagittal) as shown in Table 1, and referring to the PIML identification criteria encoded by Rosenberg et al. ${ }^{4}$, who classified PIML in scans acquired in the coronal plane as present (Fig. 1 a-c), indeterminate (Fig. 2 ab), or absent (Fig. 3 a-b), as shown in Table 2. The

Table 1. Appearance of the PIML in the different axis of examination.

\begin{tabular}{ll}
\hline Plane & Appearance \\
\hline Coronal & Two possible morphologies: 1) thick hypo-intense streak, and 2) more than two parallel hypo-intense streak \\
partially or fully viewable at the level or slightly above the PTFL. \\
Linear hypointense streak located behind the talus, starting from the lateral malleolus which runs medially in \\
continuity with the septum between the posterior tibial and flexor digitorum longus tendons. \\
Sagittal & $\begin{array}{l}\text { A) the medial part is displayed as 1) small scattered punctate formations of low signal (usually), and 2) oval } \\
\text { formation, more or less solid, dotted, located below the PITFL before the flexor hallucis longus tendon. }\end{array}$ \\
& B) the lateral part is displayed as a flattened or a nodular-like formation between PITFL and PTFL.
\end{tabular}




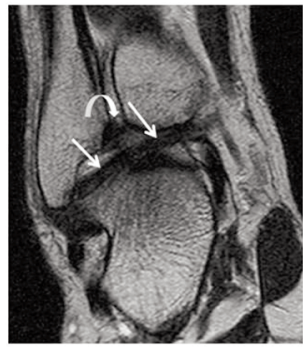

a)

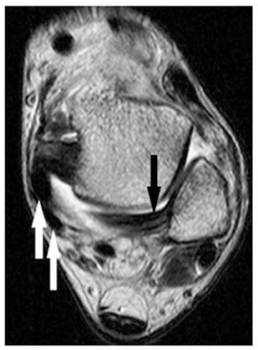

b)

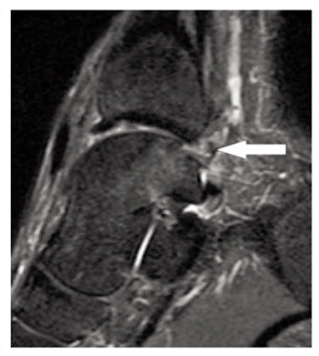

c)

Figure 1 a-c. MR scans performed with high-field scanner of posterior inter-malleolar ligament (PIML) classified as "present". a) Coronal FSE-T2 weighted scan, shows PIML as a thick hypointense string (arrows) running between fibular malleolar apex and tibial malleolus. Postero-inferior tibiofibular ligament (PITFL) (curved arrow). b) Axial FSE-T2 weighted scan, shows PIML as a linear hypointense band localized posteriorly to posterior talofibular ligament PTFL (black arrow), attached to the fibular malleolus and continuous to septum between posterior tibial and flexor digitorum longus tendons (arrows). c) Sagittal STIR scan, shows PIML as a nodular image (arrow) between PITFL and PTFL.

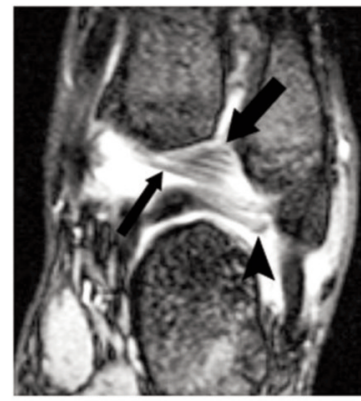

a)

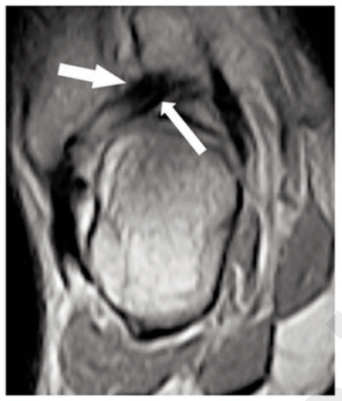

b)

Figure 2 a-b. MR scans of the posterior inter-malleolar ligament (PIML) classified as "indeterminate". a) Coronal GE2D weighted scan performed with high-field scanner shows thickened postero-inferior tibiofibular ligament (PITFL) (thick arrow) whose inferior fibers could represent PIML (thin arrow). Posterior talofibular ligament (PTFL) is also shown (arrowhead). b) Coronal GE weighted scan performed with low-field scanner shows a not well identifiable PIML (white arrow) below PITFL.

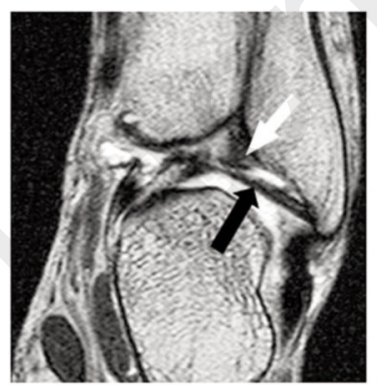

a)

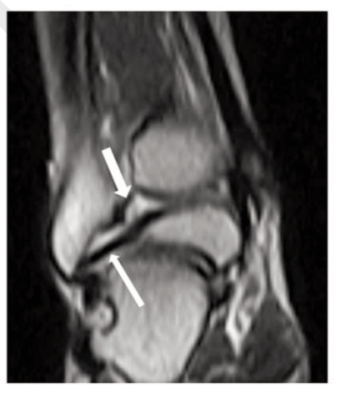

b)
Figure 3 a-b. MR scans of a posterior inter-malleolar ligament (PIML) classified as "absent". a) Coronal FSE-T2 weighted scan performed with high-field scanner shows only posterior tibiofibular ligament (PTFL) (black arrow) and postero-inferior tibio-fibular ligament (PITFL) (white arrow). b) Coronal GE weighted scan performed with lowfield scanner shows two ligaments, PITFL (thick arrow) and PTFL (long arrow).

Table 2. Classification of ligament presence.

\begin{tabular}{ll}
\hline & Definition \\
\hline Present & It was possible to identify with certainty three ligaments in the postero-lateral ligament complex: \\
the PTFL, the PITFL and PIML. & It was possible to identify with certainty three ligamentous structures in the postero-lateral ligament \\
Indeterminate & $\begin{array}{l}\text { complex but for none of them was possible to demonstrate a real insertion on both the tibial } \\
\text { malleolus or that of fibula. }\end{array}$ \\
Absent & It was only possible to identify the PTFL and PITFL. \\
\hline
\end{tabular}

images obtained with both MRI equipments (HMF and LMF), aside from evaluating the overall capability of identifying the PIML using all scan planes together, were used also to assess the probability of PIML identification using different planes, separately. The posterior ankle impingement syndrome (PAIS) was also of our interest, so the research units (two radiologists) had the mandatory to report it.

\section{Statistical Analysis}

All data were stored in an appropriate excel file. The Shapiro-Wilks test was adopted for the normality distribution. Anthropometric characteristics were compared through a t-test, in order to compare the homogeneity of groups. In order to assess the incidence for each clinical variable the frequency distribution 
Identification of normal and pathological posterior inter-malleolar ligament with dedicated high-field vs low-field MRI. A pilot study

analysis was performed. The normality distribution was set at $\mathrm{W}$ value of 0.80 . A $P$ value lower than 0.05 was considered for statistical significances. The STATISTICA Software (Tulsa, USA) 8.0 was adopted for analysis.

\section{Results}

The PIML in the HMF group has been identified with certainty in 55 patients (55\%). It was classified as "undetermined" in $28(28 \%)$ and as "absent" in the remaining $17(17 \%)$. The isolated assessment of the scans in the coronal plane has allowed the identification of PIML with certainty in all 55 cases highlighted in the overall evaluation (100\%), while the evaluation of the images on the axial and sagittal planes have allowed the identification in $37(67.27 \%)$ and 25 $(45.45 \%)$ cases respectively. In the LMF group the PIML was classified in 57 cases (57\%) as "undetermined", in $32(32 \%)$ as "absent", and has been identified with certainty only in 11 patients $(11 \%)$. The isolated assessment of the scans in the coronal plane has allowed the identification of PIML with certainty in all the 11 cases highlighted in the overall evaluation $(100 \%)$, while the evaluation of the images on the axial and sagittal planes has allowed the identification in $5(45.45 \%)$ and $3(27.27 \%)$ cases respectively. In 5 cases, 4 belonging to HFM and 1 to LMF, was highlighted a PAIS determined by the PIML with the absence of other soft tissue or bone alterations. In $100 \%$ of cases there was a thickening of the PIML and in one case there also was a synovial reaction in close contiguity with the ligamentous structures (Fig. 4). Of interest, arthroscopy was recovered from archive of 3 of the 5 cases (two exams were missing). The exams directly confirmed the role of PIML in PAIS. Results are shown in Tables 3 and 4.

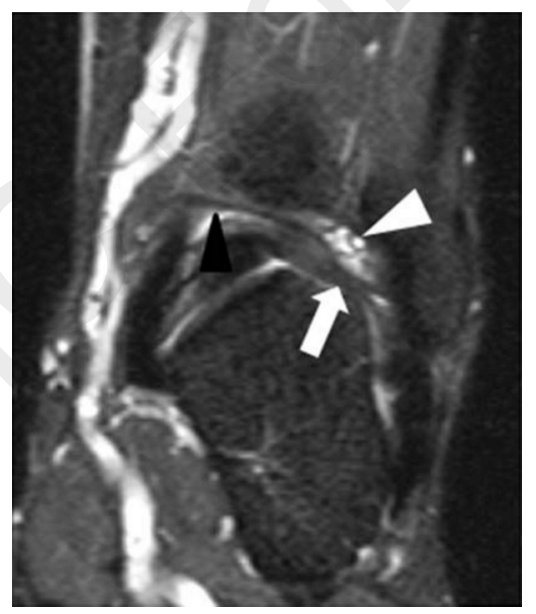

Figure 4. MR scan performed with high-field scanner (HMF) of a pathological posterior intermalleolar ligament (PIML). Coronal STIR shows thickened and inhomogeneous PIML (black arrowhead) with surrounding synovial effusions (white arrowhead). PFTL (white arrow).
Table 3. Global identification of PIML.

\begin{tabular}{lll}
\hline & HMF Group & LMF Group \\
\hline Present & $55(55 \%)$ & $11(11 \%)$ \\
Indeterminate & $28(28 \%)$ & $57(57 \%)$ \\
Absent & $17(17 \%)$ & $32(32 \%)$ \\
Total & $100(100 \%)$ & $100(100 \%)$ \\
\hline
\end{tabular}

Table 4. Identification of PIML as "present" in the single scan planes.

\begin{tabular}{lll}
\hline & HMF Group & LMF Group \\
\hline Coronal & $55(100 \%)$ & $11(100 \%)$ \\
Axial & $37(67.27 \%)$ & $5(45.45 \%)$ \\
Sagittal & $25(45.45 \%)$ & $3(27.27 \%)$ \\
Global & $100(100 \%)$ & $11(100 \%)$ \\
\hline
\end{tabular}

\section{Discussion}

In our study, the PIML has been identified with certainty in 55 cases (55\%) in HFM and in 11 cases $(11 \%)$ in LMF, and has been defined as "undetermined" in 28 cases (28\%) in HFM and in 57 cases $(57 \%)$ in LMF, and finally has been defined as "absent" in 17 cases $(17 \%)$ in HFM and in 32 cases $(32 \%)$ in LMF. The incidence of $55 \%$ in HFM we highlighted is significantly lower than those reported by Golanò et al. ${ }^{1}$, by Oh et al. $^{3}$, and by Milner and Soames ${ }^{11}$. Some explanations for these differences may be attributable to the interracial and/or anatomical variability. Oh et al. reported the midpoint thickness and width of the PIML with the average value of approximately $2.8 \mathrm{~mm}$ (range between 0.4 and 5.8 $\mathrm{mm}$ ) and $3.7 \mathrm{~mm}$ (range between 0.79 and $8.72 \mathrm{~mm})^{3}$, while Golanò et al. reported an average thickness of $2.3 \mathrm{~mm}$ (range between 1 and $5 \mathrm{~mm})^{1}$; therefore some PIML may have been unrecognized and/or were not clearly identifiable because of their small size associated with the insufficient spatial resolution of current MRI equipment. In our study we have shown that the dedicated high-field MRI compared to that at low field has allowed a greater overall capacity of identification of PIML (55\% against $11 \%$ ) and a lower percentage of doubtful cases (28\% against $57 \%$ ).

In their study, Rosenberg et al. ${ }^{4}$ reported an incidence of PIML identification in anatomical dissections of $52 \%$ (20/36 cases), whereas in MRI this identification was only $19 \%(18 / 97 \text { cases })^{4}$. Milner and Soames report an incidence of PIML identification of $72.5 \%$ (29/40 cases) $)^{11}$. Golanò et al. performed with anterior access on isolated cadaveric ankle, an incidence of $100 \%(8 / 8 \text { cases })^{1}$. Finally, Oh et al. reported an incidence of PIML identification of $81.8 \%$ in 77 dissected ankles, with perfect anatomical-MRI correlation before dissection and MRI identification of the PIML in $100 \%$ of cases $^{3}$.

The only studies in which it was reported the incidence of PIML identification with evaluation of MR images are those conducted by $\mathrm{Oh}$ et al., and Rosenberg et al. in which an incidence of $100 \%$ and $19 \%$, 
respectively ${ }^{3,4}$ has been reported. Although the investigations have been carried out both with highfield MR equipment (1.5 T), the different incidence can be perhaps justified, considering that the equipment used belongs to two different generations and the one used by $\mathrm{Oh}$ et al. is of a newer generation, and as reported by the author ${ }^{3}$ there were performed contiguous scans with the reduction of the "partial volume effect" included between the limits of the study of Rosenberg et al. ${ }^{4}$. In the evaluation of the images obtained with the dedicated low-field MR equipment with respect to those obtained with the dedicated high-field MRI we experienced greater difficulty in identifying the PIML due to the lower spatial resolution and the worse signal/noise ratio, critical factors in consideration of the size of the PIML. Other evaluation performed was on the ability of isolated images obtained with different scanning planes to permit identification of the PIML. The isolated assessment of the scans in the coronal plane has allowed the identification of the PIML with certainty in $100 \%$ of previous highlighted cases in the overall evaluation both in HFM and LMF. The isolated evaluation of the images obtained in the axial plane has allowed the identification of PIML respectively in 67.27 (HMF) and $45.45 \%$ (LMF) of cases, while the evaluation of the images obtained in the sagittal plane has allowed the identification of PIML respectively in 45.45 (HMF) and, $27 \%$ cases (LMF). In the cases where it was possible to identify the PIML as a distinct anatomical structure located in the posterior compartment of the ankle, this was identified with greater confidence in the coronal plane images in which was presented with two possible morphologies: 1) a thick hypo-intense streak or 2) as more streaks of low intensity (Fig. 5 a-b).

In our experience, moreover, the coronal scan plane was the most suitable for the detection of pathologi-

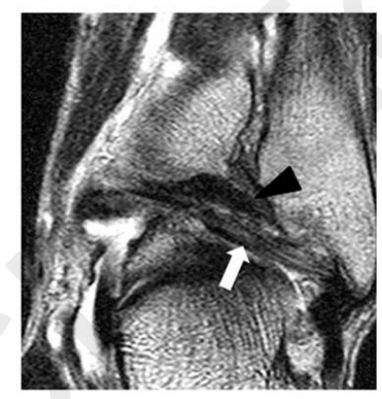

a)

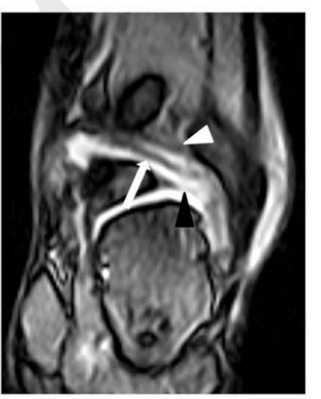

b)
Figure 5 a-b. MR scans of a posterior inter-malleolar ligament (PIML) classified as "present". a) Coronal FSE-T2 weighted image performed with HMF scanner shows PIML composed by some hypo-intense strings (white arrow) localized in the posterior region of the ankle behind the postero-inferior tibiofibular ligament (PIFTL) (black arrowhead). b) Coronal GE weighted scan performed with LMF scanner shows three ligaments, PITFL (white arrowhead), PIML (arrow), and posterior talofibular ligament (PTFL) (black arrowhead). cal changes of the signal of PIML in PAIS. In the axial images PIML was identified in fewer cases as a linear structure of low signal localized, behind the talus, starting from the lateral malleolus which runs medially in continuity with the septum between the posterior tibial tendon and the flexor digitorum longus (Fig. 1b). In the sagittal images the detection of PIML was more complex either in the identification of its medial part (Fig. 1c), which appeared as small scattered punctuate images of low signal located below the PITFL and anterior to the flexor hallucis longus tendon, and of its lateral part which looked like a flattened or a nodular image between PITFL and PTFL. Compared to the results reported by Oh et al. ${ }^{3}$ which claim the identification of the PIML in all scanning planes except, one single case in the axial plane, we have experienced greater difficulties in its detection. This difference is attributable to the increased restrictiveness of the criteria of identification that we used which determined a classification of the PIML as "indeterminate" in a fair number of cases both in HMF $(28 \%)$ and LMF $(57 \%)$. In many cases, although it was possible to visualize three-ligamentous structures in the posterior compartment of the ankle, however it was not possible to identify with certainty the peroneal or tibial insertion of the PIML and the ligamentous structures identified could also represent an accessory bundle starting from PITFL instead of the PIML.

\section{Concerns and practical implications}

As pilot study, we cannot generalise and make conclusions, but it is remarkable the fact the LMF seems to be not suitable for PIML diagnosis. We want to underline that an extensive knowledge of the anatomy of the ligamentous structures of the posterior compartment of the ankle is of fundamental importance for the recognition of the PIML and for the differential diagnosis of the causes of PAIS. Correlating MRI and arthroscopy and/or anatomical dissection can clarify the ability of MRI to identify the PIML.

\section{Conclusions}

Our study confirmed that for optimal visualization and evaluation of the PIML is required high-field MRI equipment, dedicated, or whole-body with dedicated extremity coils, as it has greater spatial resolution and better signal/noise ratio compared to dedicated low-field MRI equipment. However, even dedicated low-field MR equipment allows in a reduced number of cases to identify the PIML. In addition, the evaluation of the PIML in patients with PAIS provides an important aid in the identification of a potentially treatable cause of PAIS before performing arthroscopy of the ankle. More data with a large population scale and with a deeper statistical analysis (accuracy, power, reproducibility and tools sensibility) are needed in order to confirm this interesting pilot study. 
Identification of normal and pathological posterior inter-malleolar ligament with dedicated high-field vs low-field MRI. A pilot study

\section{Acknowledgements}

We are grateful to the DIBIMEF Department, "Section of Radiological Sciences", University of Palermo.

\section{References}

1. Golano P, Mariani PP, Rodriguez-Niedenfuhr M, Mariani PF, Ruano-Gil D. Arthroscopic anatomy of the posterior ankle ligaments. Arthroscopy. 2002;18(4):353-358.

2. Pisani G. Trattato di chirurgia del piede, 3rd edn. In: Medica M, editor. Trattato di chirurgia del piede 3rd edn. Torino: Edizioni Minerva Medica. 2004.

3. Oh CS, Won HS, Hur MS, et al. Anatomic variations and MRI of the intermalleolar ligament. AJR Am J Roentgenol. 2006; 186(4):943-947.

4. Rosenberg ZS, Cheung YY, Beltran J, Sheskier S, Leong M, Jahss M. Posterior intermalleolar ligament of the ankle: normal anatomy and MR imaging features. AJR Am J Roentgenol. 1995;165(2):387-390.

5. Stoller DW, Ferkel RD, Beltran S. The ankle and foot. Magnetic resonance imaging in orthopaedics and sports medicine $3 r d$ edn. Philadelphia, PA: Lippincott, Wilkins and Williams. 2007.

6. Hamilton WG. Foot and ankle injuries in dancers. Clin Sports Med. 1988;7(1):143-173.

7. Lewis OJ. The joints of the evolving foot. Part I. The ankle joint. J Anat. 1980;130(Pt 3):527-543.

8. Fiorella D, Helms CA, Nunley JA, 2nd. The MR imaging features of the posterior intermalleolar ligament in patients with posterior impingement syndrome of the ankle. Skeletal Radiol. 1999;28(10):573-576.

9. Cerezal L, Abascal F, Canga A, et al. MR imaging of ankle impingement syndromes. AJR Am J Roentgenol. 2003; 181(2):551-559.

10. Robinson $P$, White LM. Soft-tissue and osseous impingement syndromes of the ankle: role of imaging in diagnosis and management. Radiographics. 2002;22(6):1457-1469; discussion 70-71.

11. Milner CE, Soames RW. Anatomy of the collateral ligaments of the human ankle joint. Foot Ankle Int. 1998;19(11):757-760.

12. Morgan CD. Gross and arthroscopic anatomy of the ankle. In: McGinty J, editor. Operative arthroscopy. Philadelphia, PA: Lippincott.Raven. 1996;1101-1117.

13. Padulo J, Oliva F, Frizziero A, Maffulli N. Muscle, Ligaments and Tendons Journal. Basic principles and recommendations in clinical and field science research. MLTJ. 2013;4:250-252. 